\title{
Factores críticos de éxito para la implementación de Business Process Management (BPM): estudio de caso para la cadena de suministro de una empresa del sector floricultor
}

\author{
Alexander Gutiérrez Sánchez ${ }^{1}$ \\ Corporación Universitaria Minuto de Dios - UNIMINUTO \\ algutierrez@uniminuto.edu.co \\ Claudia Rodríguez Ríos ${ }^{2}$ \\ Escuela Colombiana de Ingeniería Julio Garavito \\ claudia.rodriguez@escuelaing.edu.co \\ Andrés Felipe Santos Hernández ${ }^{3}$ \\ Escuela Colombiana de Ingeniería Julio Garavito \\ andres.santos@escuelaing.edu.co
}

Fecha de recepción: 03 de mayo de 2018

Fecha de aprobación: 09 de septiembre de 2018

DOI: https://doi.org/10.21158/01208160.n0.2018.2019

Cómo citar este artículo / To reference this article / Comment citer cet article / Para citar este artigo:

Gutiérrez Sánchez, A.; Rodríguez Ríos, C. y Santos Hernández, A. F. (2018). Factores críticos de éxito para la implementación de Business Process Management (BPM): estudio de caso para la cadena de suministro de una empresa del sector floricultor. Revista EAN, Edición especial, pp 85-108. DOI: https://doi.org/10.21158/01208160.n0.2018.2019

\section{- Resumen}

Se diseñó un modelo que muestra la interacción entre los factores críticos de éxito (FCE) para implementar Business Process Management (BPM) y los procesos de la cadena de suministro a través de cada una de las fases de implementación, se identificaron aquellos FCE más relevantes a través de un análisis de la literatura y se validaron por un grupo de expertos. Luego se utilizó un enfoque de estudio de caso en un cultivo de flores ubicado en la sabana de Bogotá para validar empíricamente el modelo propuesto. Los resultados se convirtieron en un diagnóstico que guía a esta empresa sobre los aspectos prioritarios para mejorar y poder dirigir sus esfuerzos hacia estos en particular, asegurando un mayor grado de éxito en la implementación de BPM en este proceso. El modelo establecido propone un marco de referencia para futuras investigaciones que permitan hacer un diagnóstico y determinar el nivel en el que se encuentre la empresa de acuerdo con los FCE propuestos.

\section{Palabras Clave}

Dirección de proyectos, cadenas de suministro, floricultura - estudio de casos, gestión industrial, control de la producción.

\footnotetext{
${ }^{1}$ Magister en Ingeniería Industrial de la Escuela Colombiana de Ingeniería Julio Garavito, Ingeniero Industrial de la misma universidad y Especialista en Gerencia en Logística Integral de la Universidad Militar Nueva Granada. Profesor de planta de la Corporación Universitaria Minuto de Dios - UNIMINUTO

${ }^{2}$ Magister en Ingeniería Industrial de la Pontificia Universidad Javeriana Bogotá, Especialista en Desarrollo y Gerencia Integral de Proyectos, e Ingeniera de Sistemas de la Escuela Colombiana de Ingeniería Julio Garavito. Profesora de planta del Programa de Ingeniería Industrial de La Escuela Colombiana de Ingeniería Julio Garavito.

${ }^{3}$ MBA de la Universidad el Rosario, Ingeniero industrial de la Escuela Colombiana de Ingeniería Julio Garavito..Profesor de planta del Programa de Ingeniería Industrial de La Escuela Colombiana de Ingeniería Julio Garavito.
} 


\section{Success critical factors for the implementation of Business Process Management (BPM): a case study for the supply chain in a flower company}

Abstract. A model which showed interaction between success critical factors (SCF) was designed to implement Business Process Management (BPM) and the processes involved in the supply chain through the stages of implementation, which finally identified the most relevant SCF factors by the revision of existing literature and validation made by a group of experts. Next, a case study focus was used in flower crops located in Sabana de Bogota area to empirically validate the proposed model. Its outcomes became a diagnosis guideline for this company to determine the relevant aspects to be considered when improving and conducting efforts to achieve them, also assuring a higher level of success in the implementation of BPM processes. This established model proposes a referential framework for future investigations which will allow to diagnose and determine the level of development the company reaches based on SCF factors already established.

key words: Project management, supply chain, Flower crops- case study, industrial management, production quality control.

\section{Facteurs critiques pour la réussite de la mise en place du Business Process Management (BPM): étude de cas pour la chaîne d'approvisionnement d'une entreprise du secteur de la floriculture}

Résumé. Cet article a pour objectif de montrer l'interaction des facteurs clés de succès (FCS) lors de la mise en cuvre de la gestion des processus métier (GPM) et des processus de la chaîne d'approvisionnement à travers chacune des phases d'implémentation pour identifier les FCS les plus pertinents via une analyse de la littérature validée par un groupe d'experts. Une étude de cas a ensuite été réalisée dans une entreprise floricole située non loin de Bogotá afin de valider empiriquement le modèle objet de cette étude. Les résultats ont permis un diagnostic aidant l'entreprise à hiérarchiser les secteurs à améliorer en priorité et à orienter ses efforts vers ceux-ci afin d'assurer un plus grand succès dans la mise en œuvre des GPM dans ce processus. Le modèle établit propose un cadre pour la recherche future permettant de réaliser un diagnostic et de déterminer le niveau auquel se situe la société selon les FCS proposés.

Mots clefs: Gestion de projets, chaînes d'approvisionnement, floriculture - études de cas, gestion industrielle, contrôle de production.

\section{Fatores críticos de sucesso para a implementação de Business Process Management (BPM): estudo de caso sobre a cadeia de fornecimento de uma empresa do setor floricultor}

Resumo. Desenhou-se um modelo que mostra a interação entre os fatores críticos de sucesso (FCE) para implementar Business Process Management (BPM) e os processos da cadeia de fornecimento através de cada uma das fases de implementação. Identificaram-se aqueles FCE mais relevantes através de uma análise da literatura e validaram-se os dados por um grupo de peritos. Depois utilizou-se um enfoque de estudo de caso num cultivo de flores localizado ao na savana de Bogotá para validar empiricamente o modelo proposto. Os resultados se tornaram um diagnóstico que guia a esta empresa sobre os aspectos prioritários para melhorar e poder dirigir seus esforços neste sentido, assegurando um maior grau de sucesso na implementação de BPM. O modelo estabelecido propóe um marco de referência para futuras pesquisas que permitam fazer um diagnóstico e determinar o nível no qual se encontre a empresa de acordo com os FCE propostos.

Palavras-chave: Direção de projetos, cadeia de fornecimento, floricultura - estudo de casos, gerenciamento industrial, controle da produção. 


\section{Introducción}

$\mathbf{E}_{\mathrm{p}}^{1}$ 1 Business Process Management (BPM) ha probado ser una herramienta que conlleva el mejoramiento del desempeño y la competitividad empresarial, y los procesos de la cadena de suministro no son ajenos a estos avances. El BPM es un sistema de gestión basado en procesos que facilita la estandarización, la documentación, la automatización y el control de estos, que mejora la competitividad y la innovación de las organizaciones (Dumas, La Rosa, Mendling y Reijers, 2013; Rodríguez, 2015). El éxito en la implementación del BPM en las empresas depende de diferentes factores (Bandara, Alibabaei y Aghdasi, 2009), y es con frecuencia una tarea compleja y poco exitosa en la cual se consumen recursos y se requiere el compromiso por parte de todos los implicados (Buh, Kovačič y Indihar Štemberger, 2015).

Ravesteyn y Batenburg (2010) y Trkman, McCormack, Valadares y Bronzo (2010) argumentan que lo anterior es la causa por la cual hoy en día existen elevados índices de fracaso en implementaciones del BPM, lo que impulsa la investigación relacionada con los factores críticos de éxito (FCE). Así mismo, la creciente aplicación del BPM en diferentes escenarios empresariales involucra nuevos requisitos en cuanto a sus métodos y prácticas, que el conocimiento actual no cubre de manera definitiva, por lo que, si se desea una implementación exitosa, se deben tener en cuenta las condiciones específicas de la organización, por ende, los profesionales deben ser capaces de identificar características relevantes para entender el contexto en el que se desarrolla una iniciativa del BPM (Vom Brocke, Schmiedel y Zelt, 2015a).

La revisión bibliográfica sugiere que, a pesar de que el concepto BPM ha sido bastante estudiado, aún no cuenta con una base teórica apropiadamente fundamentada sobre el proceso de implementación en las organizaciones (Trkman, 2010). De igual manera, la literatura ofrece conceptos generales acerca de los FCE y el papel que desempeñan en la implementación de sistemas BPM (Bandara, Gable y Rosemann, 2005), por lo que en el presente trabajo se busca identificar y estudiar estos FCE y su interrelación, tras lo cual se llega a desarrollar un modelo para la cadena de suministro de una empresa del sector floricultor. Para este fin, se estructuró un estudio de caso desarrollado en un cultivo de flores ubicado en la sabana de Bogotá para identificar cuáles son los FCE más relevantes que interactúan en cada uno de estos procesos y a través de las fases de implementación del BPM.

El artículo está estructurado así: en la siguiente sección, se presenta la revisión de la literatura que incluye la identificación de los FCE, se describe a continuación la metodología y la presentación del estudio de caso. Posteriormente, se muestran los resultados y la discusión, para finalizar con las conclusiones y los futuros trabajos sobre esta temática.

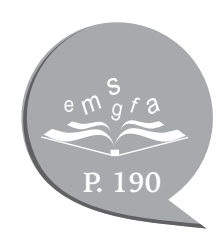




\section{Revisión de la literatura}

$\mathrm{D}$ esde finales del siglo XX se han investigado diferentes tendencias relacionadas con temas gerenciales encaminados a mejorar la gestión y la operación en las empresas, como el Supply Chain Management (SCM), el Customer Relationship Management (CRM), el Total Quality Management (TQM), el Knowledge Management (KM), el Supply Chain Integration (SCI) (Bandara, Gable y Rosemann, 2005; Palma-Mendoza, Neailey y Roy, 2014), de modo que estos son los más utilizados.

Por otra parte, se encuentran estudios relacionados con el Business Process Reengineering (BPR), el Business Process Orientation (BPO), el Lean Manufacturing, Six sigma y el Balance Score Card (BSC) (Asikhia y Awolusi, 2015; Hammer, 2002; McCormack y Johnson, 2001; Ozcelik, 2010; Rodríguez, 2015; Škrinjar y Trkman, 2013; Trkman et al., 2010), todos enfocados en el mejoramiento del desempeño empresarial, donde se distingue que el BPM es el concepto predominante (Wong, 2013).

En este contexto, se hallaron investigaciones referentes a herramientas y técnicas de modelamiento de procesos (Bandara, Gable y Rosemann, 2005), estandarización de procesos y su impacto sobre los procesos de negocio (Münstermann, Eckhardt y Weitzel, 2010), evaluación del impacto que tienen los FCE sobre el desempeño de las compañías en la ejecución de proyectos BPR (Asikhia y Awolusi, 2015) y el diseño de estructuras metodológicas para la implementación de modelos BPM (Batocchio, Ghezzi y Rangone, 2016; Hernández-Nariño, Delgado-Landa, Marqués-León, Nogueira-Rivera, Medina-León y Negrín-Sosa, 2016). Otras investigaciones abordan temas concernientes a la definición de planes estratégicos y cómo el BPM puede apoyar no solo su construcción, sino también garantizar su comunicación a todos los niveles de la organización. Para ello, se utilizan modelos de ciclo de vida del BPM (Morais, Kazan, De Pádua y Costa, 2010) o se apoyan en la dinámica de sistemas para analizar la relación que la empresa tiene con su entorno (Munive-Hernández, Dewhurst, Pritchard y Barber, 2004).

A pesar de esto, algunos autores sugieren que no existen suficientes trabajos prácticos que muestren la evaluación de los FCE en la implementación del BPM, argumentando que el éxito de la implementación varía según la unidad en la que se desarrolle, así como del tiempo para el que se establecen los objetivos (Bandara, Gable y Rosemann, 2005; Trkman, 2010).

Algunas revisiones de la literatura sugieren la necesidad relevante de explicar las interacciones existentes entre los procesos de negocio, las inversiones en tecnología, el mejoramiento continuo, la cultura, entre otros, y deben identificarse cuáles son los factores más notables para el éxito de la implementación del BPM en las empresas (Bandara, Gable y Rosemann, 2005, 2006; Munive-Hernández et al., 2004; Škrinjar y Trkman, 2013; Trkman, 2010).

\subsection{Competitividad y gestión de la cadena de suministro}

Porter (1985) desarrolló el concepto de competitividad en la década de 1980 y explica que las alternativas estratégicas de una empresa están limitadas por el entorno del mercado que disputan; pero, si el sector no es competitivo, es difícil que una empresa logre serlo. Una manera sistemática de estudiar las actividades que desarrolla una compañía y cómo éstas 
interactúan, es analizando las fuentes de su ventaja competitiva y una herramienta para hacerlo es la cadena de valor.

La cadena de valor es un conjunto de procesos estructurados, cuyo objetivo es brindar una propuesta de valor a los clientes y generar valor económico para todos los implicados, identificando formas de generar mayores beneficios para el consumidor (Porter, 1985). La cadena de valor de una organización hace parte de un largo flujo de actividades que Porter denominó sistema de valor, en otras palabras, una cadena de suministro. La premisa es que dicha ventaja puede darse a partir de varias fuentes y una de ellas es la gestión de la cadena de suministro (Supply Chain Management [SCM]) (Crhistopher, 2011; Min y Mentzer, 2004).

Aunque algunos autores afirman que, no existe una definición universal de la SCM, varias perspectivas de académicos e involucrados desde la práctica toman el Supply Chain Operations Reference Model (SCOR-model) como una conceptualización razonable de la SCM, que es ampliamente citado por diferentes academias (Esper, Defee y Mentzer, 2010; Jüttner, Christopher y Godsell, 2010; McCormack y Johnson, 2001). Sin embargo, se puede decir que la SCM es la coordinación sistémica y estratégica de las funciones tradicionales del negocio en la compañía, así como de los procesos a través de la cadena de suministro, cuya finalidad es mejorar a largo plazo el funcionamiento individual, tanto de la compañía como de todo el sistema al que pertenece (Mentzer et al., 2001).

Desde este punto de vista, es necesaria una integración total que permita modificar las prácticas tradicionales por funciones a un desempeño enfocado en procesos, razón por la que se requieren nuevas prácticas de colaboración dirigidas a administrar eficien- temente los procesos de la cadena de suministro, haciendo hincapié en la generación de valores que ayuden a entender cómo la organización debería gerenciar estratégicamente su cadena y la influencia que puede llegar a tener la orientación por procesos sobre la SCM para generar una verdadera ventaja competitiva (Esper, Defee y Mentzer 2010; McCormack y Johnson, 2001).

Es claro que la competitividad empresarial está íntimamente ligada a la gestión estratégica de los procesos. Comprender que la integración de todos los eslabones de la cadena de suministro desempeña un papel trascendental en la satisfacción del cliente y de la generación de valor para todos los implicados es la clave para la ventaja competitiva de todo el sistema.

\subsection{Business Process Management}

Es importante resaltar que bajo la orientación por procesos los responsables se enfocan primordialmente en el desempeño de los procesos de inicio a fin y en alinearlos con la estrategia organizacional, con los objetivos estratégicos y con la creación de valor al cliente (Ceribeli, Dallavalle de Pádua y Merlo, 2013), razón por la que el BPM va más allá del análisis, el diseño, el desarrollo y la ejecución de los procesos de negocio, incluso la interacción, el control y la optimización de estos (Kohlbacher y Gruenwald, 2011).

Dado esto, el BPM es un enfoque estructurado que sirve para analizar y mejorar permanentemente actividades fundamentales de la organización en las que existe un gran valor agregado (Zairi, 1997). Además, utiliza métodos, técnicas y software para diseñar, analizar y controlar procesos operacionales en los que se incluyen personas, aplicaciones, documentos $\mathrm{u}$

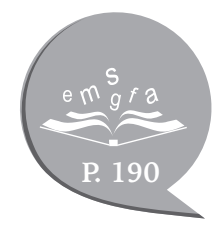


otras formas de información para apoyar los procesos de negocio de las organizaciones (Ghalimi y McGoveran, 2004).

Armistead, Pritchard y Machin (1999) afirman que el BPM se está convirtiendo en un componente importante para la gestión de la compañía a partir de la adopción de buenas prácticas desarrolladas en las diferentes áreas. Dichas prácticas también deben adoptar un enfoque más estratégico que beneficiará el desempeño de toda la organización, la cual obtendrá una mejor productividad, reducirá los tiempos de respuesta al cliente y los costos, y generará mayor valor, no solo para la compañía, sino para toda la cadena de suministro (Münstermann, Eckhardt y Weitzel, 2010).

Se puede ver que, aunque los beneficios del BPM son claramente entendidos, algunos autores expresan que existe ambigüedad sobre el concepto y carencia de aplicaciones en contextos empresariales (Ariyachandra y Frolick, 2008; Bider y Perjons, 2015; Vom Brocke, Zelt y Schmiedel, 2015b). Según la Association of Business Process Management Professionals International (ABPMP), «el BPM es un método que sirve para identificar, diseñar, ejecutar, documentar, medir, monitorear, controlar y mejorar procesos de negocio, sean estos automatizados o no, para alcanzar los resultados de la estrategia organizacional» (Antonucci, Bariff, Benedict, Champlin, Downing, Franzen, Madison, Lusk, Spanyi, Treat, Zhao, \& Raschke, 2009).

En ocasiones, el término es identificado como gestión del desempeño empresarial con dos objetivos: a) facilitar la creación de las metas estratégicas y desarrollar objetivos específicos, así como los correspondientes indicadores útiles para toda la empresa, y b) dar soporte al desempeño de esos objetivos que se asocian con las métricas operacionales (Ariyachandra y Frolick, 2008).
Otros autores tratan el término procesos de negocio desde dos perspectivas: a) tipo de proceso de negocio, entendido como una "plantilla" con la que gestionar diferentes escenarios de negocio, b) instancias del proceso de negocio, que es el escenario gestionado de acuerdo con la plantilla sugerida (Bider y Perjons, 2015).

El BPM es un sistema integrado de gestión basado en procesos que utiliza sistemas de información especializados y cubre todos los procesos operativos y de negocio de la organización para hacerla más productiva y competitiva, asegurando un mejoramiento continuo y un mantenimiento permanente de los procesos acorde con la estrategia de la empresa (Rodríguez, 2015).

También el BPM puede entenderse como un conjunto de herramientas y métodos empleados para soportar el diseño, el desempeño, la administración y el análisis de los procesos (Bosilj, Milanović y Suša, 2015), lo que exige un alto grado de compromiso por parte de toda la compañía, el cual se dirige con la implementación y gestión de un modelo, cuyas fases se encuentran bien definidas y establecen lineamientos para la organización, fundamentando las bases para el proceso de mejoramiento continuo (Dallavalle, Hornos, Segatto, De Souza y Jabbour, 2014).

Teniendo presente la revisión, el BPM es una herramienta de gestión basada en procesos enfocados en el cliente y que mejora el desempeño de las compañías, no solo desde el punto de vista de los costos, sino que su implementación genera un entorno empresarial efectivo que refleja alineación entre los procesos y la estrategia organizacional, con personas comprometidas y productos -bienes y servicios- de calidad, satisfaciendo eficazmente el mercado, lo que a su vez llevará a mejorar la eficiencia, la competitividad y la innovación empresarial. 


\subsection{Factores críticos de éxito (FCE)}

Un factor es crítico cuando es indispensable para el cumplimiento de las metas empresariales. Desde el punto de vista del BPM, un FCE se basa en la idea de que ciertos aspectos son esenciales para asegurar la culminación de los proyectos de implementación del BPM, entendiendo como «implementación exitosa» el cumplimiento permanente de las metas previamente determinadas en el proyecto (Trkman, 2010), el cumplimiento satisfactorio de los objetivos propuestos para el proyecto (Bandara, Alibabaei y Aghdasi, 2009) o la medida a través de modelos de madurez que describen el nivel de desarrollo del BPM en las empresas donde está implementado (Buh, Kovačičy Indihar, 2015; Škrinjar y Trkman, 2013).
De acuerdo con la revisión de la literatura, se encuentran trabajos con conceptos similares acerca de los FCE que afectan la implementación del BPM (Ariyachandra y Frolick, 2008). Autores como Bandara, Gable y Rosemann (2005), Škrinjar y Trkman (2013) y Trkman (2010) argumentan que algunos estudios se limitan solo a la identificación de FCE, en los que pocas veces se muestran pruebas experimentales que lleven a las empresas a una implementación exitosa del BPM, y aunque muchos autores han admitido consideraciones más holísticas de los FCE, aún existen diferentes percepciones del papel que juegan otros factores en la implementación del BPM, como la cultura y las personas (Schmiedel, Vom Brocke y Recker, 2014) (tabla 1).

Tabla 1. Factores críticos de éxito

\begin{tabular}{|c|c|}
\hline FCE estudiados/propuestos & Autor(es) \\
\hline $\begin{array}{l}\text { Alineación estratégica, Key } \\
\text { Performance Indicator (KPI), } \\
\text { dirección empresarial, enfoque al } \\
\text { cliente }\end{array}$ & $\begin{array}{l}\text { Bai y Sarkis, 2013; Bandara et al., 2009; Buh et al., 2015; Ceribeli et al., 2013; } \\
\text { Imanipour, Talebi y Rezazadeh, 2012; Ravesteyn y Batenburg, 2010; Rosemann y } \\
\text { Vom Brocke, 2010; Škrinjar y Trkman, 2013; Trkman, 2010; Vom Brocke, Schmiedel } \\
\text { et al., 2015)research has shown that as many as } 60-80 \% \text { of BPM initiatives are } \\
\text { unsuccessful. This study provides a methodology to evaluate BPM implementation } \\
\text { critical success factors (CSFs }\end{array}$ \\
\hline Recursos financieros & $\begin{array}{l}\text { Ahmad, Francis y Zairi, 2007; Ariyachandra y Frolick, 2008; Burlton, 2011; } \\
\text { Imanipour et al., 2012; Ohtonen y Lainema, } 2011\end{array}$ \\
\hline $\begin{array}{l}\text { Gestión del conocimiento, } \\
\text { capitalización del conocimiento }\end{array}$ & $\begin{array}{l}\text { Burlton, 2011; Mircea, Ghilic-Micu, Stoica y Sinioros, 2016; vom Brocke, Schmiedel } \\
\text { et al., } 2015\end{array}$ \\
\hline $\begin{array}{l}\text { Tecnología de la información (TI), } \\
\text { automatización, digitalización, } \\
\text { recursos de TI }\end{array}$ & $\begin{array}{l}\text { Ahmad, Francis y Zairi (2007), Al-Mashari y Zairi (1999), Asikhia y Awolusi } \\
\text { (2015), Bai y Sarkis (2013), Bandara, Alibabaei y Aghdasi (2009), Imanipour, } \\
\text { Talebi y Rezazadeh (2012), Ohtonen y Lainema (2011), Ravesteyn y Batenburg } \\
\text { (2010), Rodríguez, Pérez y Rodríguez (2015), Rosemann y Vom Brocke (2010), } \\
\text { Śkrinjar y Trkman (2013) y Trkman (2010) }\end{array}$ \\
\hline $\begin{array}{l}\text { Estructura organizacional, tamaño de } \\
\text { la empresa, procesos }\end{array}$ & $\begin{array}{l}\text { Asikhia y Awolusi (2015), Burlton (2011), Imanipour, Talebi y Rezazadeh (2012), } \\
\text { Mircea et al. (2016), Ravesteyn y Batenburg (2010), Rodríguez, Pérez y Rodríguez } \\
\text { (2015), Škrinjar y Trkman (2013), Trkman (2010) y Vom Brocke, Zelt y Schmiedel } \\
\text { (2015b) }\end{array}$ \\
\hline $\begin{array}{l}\text { Personas (perfil, competencias, } \\
\text { capacitación) }\end{array}$ & $\begin{array}{l}\text { Ahmad, Francis y Zairi (2007), Al-Mashari y Zairi (1999), Bandara, Gable y } \\
\text { Rosemann (2005), Box y Platss (2005), Buh, Kovačič y Indihar (2015), Ceribeli, } \\
\text { Dallavalle y Merlo (2013) y Rodríguez, Pérez y Rodríguez (2015). }\end{array}$ \\
\hline
\end{tabular}


Tabla 1. Factores críticos de éxito (Continuación)

\begin{tabular}{|c|c|}
\hline FCE estudiados/propuestos & Autor(es) \\
\hline Trabajo en equipo, empoderamiento & $\begin{array}{l}\text { Ahmad, Francis y Zairi (2007), Ariyachandra y Frolick (2008), Bai y Sarkis (2013), } \\
\text { Bandara, Gable y Rosemann (2005), Imanipour, Talebi y Rezazadeh (2012), } \\
\text { Ohtonen y Lainema (2011), Škrinjar y Trkman (2013) y Trkman (2010) }\end{array}$ \\
\hline $\begin{array}{l}\text { Liderazgo, apoyo y participación de la } \\
\text { alta gerencia }\end{array}$ & $\begin{array}{l}\text { Ariyachandra y Frolick (2008), Asikhia y Awolusi (2015), Bai y Sarkis (2013), } \\
\text { Bandara, Gable y Rosemann (2005), Box y Platss (2005), Buh, Kovačič y Indihar } \\
\text { (2015), Ceribeli, Dallavalle y Merlo (2013), Ohtonen y Lainema (2011) y } \\
\text { Ravesteyn y Batenburg (2010) }\end{array}$ \\
\hline Gestión de proyectos & $\begin{array}{l}\text { Ahmad, Francis y Zairi (2007), Al-Mashari y Zairi (1999), Asikhia y Awolusi } \\
\text { (20159, Bai y Sarkis (2013), Bandara, Alibabaei y Aghdasi (2009), Box y Platss } \\
\text { (2005), Ohtonen y Lainema (2011) y Ravesteyn y Batenburg (2010) }\end{array}$ \\
\hline Entorno, competencia, competitividad & $\begin{array}{l}\text { Box y Platss (2005), Imanipour, Talebi y Rezazadeh (2012), Rodríguez, Pérez y } \\
\text { Rodríguez (2015) y Vom Brocke, Zelt y Schmiedel (2015b) }\end{array}$ \\
\hline Gestión del cambio, cultura & $\begin{array}{l}\text { Ahmad, Francis y Zairi (2007), Al-Mashari y Zairi (1999), Ariyachandra y Frolick } \\
\text { (2008), Asikhia y Awolusi (2015), Bai y Sarkis (2013), Bandara, Alibabaei y } \\
\text { Aghdasi (2009), Bosilj et al. (2015), Box y Platss (2005), Buh, Kovačič y Indihar } \\
\text { (2015), Imanipour, Talebi y Rezazadeh (2012), Mircea, Ghilic-Micu, Stoica y } \\
\text { Sinioros (2016), Ohtonen y Lainema (2011), Ravesteyn y Batenburg (2010), } \\
\text { Rodríguez, Pérez y Rodríguez (2015) y Trkman (2010) }\end{array}$ \\
\hline $\begin{array}{l}\text { Comunicación, calidad de la } \\
\text { comunicación, confianza }\end{array}$ & $\begin{array}{l}\text { Ahmad, Francis y Zairi (2007), Ariyachandra y Frolick (2008), Bandara, Alibabaei } \\
\text { y Aghdasi (2009), Box y Platss (2005), Burlton (2011), Imanipour, Talebi y } \\
\text { Rezazadeh (2012), Ohtonen y Lainema (2011), Ravesteyn y Batenburg (2010) y } \\
\text { Rosemann y Batenbur (2010) }\end{array}$ \\
\hline Mejoramiento continuo, innovación & $\begin{array}{l}\text { Ahmad, Francis y Zairi (2007), Asikhia y Awolusi (2015), Burlton (2011), Ohtonen } \\
\text { y Lainema (2011), Ravesteyn y Batenburg (2010) y Trkman (2010) }\end{array}$ \\
\hline
\end{tabular}

Fuente. Elaboración propia.

\section{Metodología}

Esta investigación se desarrolló en el periodo comprendido entre enero y octubre de 2017 en una empresa dedicada al cultivo y la exportación de rosas ubicada en la sabana de Bogotá. Se realizaron visitas al cultivo, en las que, a través de observación directa, entrevistas semiestructuradas y consulta de documentos, se identificó la cadena de suministro y se levantó información acerca de sus procesos para construir su cadena de valor y su Value Stream Mapping (VSM).
Inicialmente, se identificaron los FCE según la revisión de la literatura, tomando diferentes investigaciones y estudios de caso relacionados con implementaciones del BPM. Luego de clasificarlos, se expusieron a un grupo de expertos, quienes discutieron desde su experiencia para refinarlos y después llevarlos a un workshop con el gerente de la empresa objeto de estudio, quien además solicitó mantener en confidencialidad su razón social, así como la información utilizada para llevar a cabo el estudio. 


\section{Estudio de caso}

C olombia posee algunas de las mejores tierras cultivables para flores tipo exportación. Cuenta con más de 7000 ha de producción, de las que Cundinamarca posee el $72 \%$, donde la mayor concentración de cultivos se ubica en la sabana de Bogotá, Antioquia tiene el 27 $\%$ y centro-occidente el $1 \%$. De acuerdo con la Asociación Colombiana de Exportadores de Flores (Asocolflores), entre 2012 y 2016, el área destinada para el cultivo de flores aumentó un $18 \%$ alcanzando una producción de 225 $000 \mathrm{t}$, lo que representa un total de más de USD 1100 millones en ventas (Asocolflores, 2017). Además, el sector floricultor aporta en promedio 14 puestos de trabajo por hectárea que representan más de 130000 empleos formales, entre directos e indirectos, en los que el $65 \%$ de los trabajadores son mujeres, muchas de ellas madres cabeza de familia, de modo que es la actividad agrícola que más empleos genera por unidad de área (Asocolflores, 2017), convirtiéndolo en un agente de desarrollo para el país.

Colombia exporta a más de 80 países, cuyo principal destino son los Estados Unidos, a los que se envía el $75 \%$ de la producción de rosas equivalentes a 141700 t. El segundo comprador es la Unión Europea con el 9,8 \% de las exportaciones, el Reino Unido con un $5 \%$, mientras que a Canadá, Japón, Rusia y Holanda con el $3 \%$ cada uno (Asocolflores, 2017). Esto quiere decir que alrededor de 225 000 t de flores fueron exportadas en 2016, lo que convierte a este producto en el segundo más importante para los ingresos del país (Asocolflores, 2017). En comparación, para el primer semestre de 2017, se exportaron 129000 t de flores que representan USD 785 millones, es decir, un crecimiento del 3,5\% en volumen y del $8 \%$ en valor, frente al mismo periodo de
2016 (Departamento Administrativo Nacional de Estadística -DANE, 2017).

Aunque este sector es uno de los más importantes para Colombia, la producción de flores es una actividad intensiva en mano de obra y poca apropiación de la gestión por procesos. Pese a esto, en el transcurso de más de cincuenta años, se ha logrado posicionar como el segundo mayor exportador de flores frescas cortadas (Asocolflores, 2017), lo que explica su peso en la balanza comercial. Es importante mencionar que, desde su inicio, el objetivo del sector floricultor ha sido la exportación, razón por la cual lo ha llevado a mejorar no solo sus procesos productivos y comerciales, sino también aquellos que componen su cadena de abastecimiento, con el fin de mantener su competitividad y ratificar su presencia en los mercados internacionales.

De acuerdo con Quirós (2001), se entiende que «las condiciones para garantizar el mercado internacional están ligadas a contactos en la cadena productiva completa" (p. 64), lo que pone en evidencia que los empresarios deben adelantar acciones, no solo en temas comerciales, sino en otros aspectos, incluidos, por ejemplo, la evaluación de la eficiencia en sus procesos o la forma y velocidad en el intercambio de información, de tal modo que los prepare para enfrentar la competencia proveniente de otros países. En general, las empresas colombianas no cuentan con procesos estandarizados, carecen de recursos tecnológicos, los sistemas de información no están enfocados en la gestión por procesos, los sistemas no se adaptan a los negocios y sus particularidades, y otros casos en los que, incluso, las organizaciones no tienen indicadores de gestión que permitan medir su desempeño (Galvis-Lista y GonzálezZabala, 2014). 
Particularmente, en el sector floricultor, algunos cultivos han trabajado para la integración de sus diferentes áreas funcionales; sin embargo, existen casos en los que, por una parte, no hay información adecuada o se carece de un esquema de actualización para su efectivo funcionamiento, y por otra, en los cultivos de flores hay poco personal administrativo, lo que conlleva que los sistemas puntuales o incluso aquellos que han tratado de fortalecerse a través de la integración de procesos no sean administrados con una visión corporativa clara (González, 2013; Pantoja y Donoso, 2015).

Acorde con la revisión de la literatura, se evidencian pocas relaciones con el tema de estudio. Específicamente hablando del sector floricultor, se hallan los realizados por Quirós (2001), González (2013), González y Sarmiento (2014), Sepúlveda (2014) y Pantoja y Donoso (2015), quienes analizan diferentes aspectos relacionados con la floricultura colombiana, pero en ninguno de estos trabajos hay referencias sobre la identificación de los FCE, para las implementaciones del BPM, iniciativas de la BPR o temas relacionados, que estén dirigidos a mejorar la gestión por procesos en empresas floricultoras, razón por la cual se desea indagar perspectiva y se pretende identificar cuáles son aquellos FCE relevantes en proyectos de implementación del BPM, según las características de la cadena de suministro de una empresa del sector floricultor, para lo cual se desarrolló un estudio de caso.

Un estudio de caso es pertinente cuando el investigador tiene poco o ningún control sobre las situaciones por estudiar y es de utilidad cuando se desean responder preguntas acerca de cómo o por qué sucede algo. Con el propósito de identificar, documentar y divulgar aquellos
FCE que afectan la implementación del BPM, en el presente artículo se examina a profundidad un cultivo de rosas, cuyas unidades de análisis son los procesos de su cadena de suministro, lo que corresponde a un caso simple de diseño incrustado, es decir, un caso tipo II (Yin, 2003).

Además, se encuentran en la literatura múltiples beneficios al adoptar el BPM, por lo que plantear estudios de caso da la posibilidad a otros investigadores de tener una mayor comprensión de temas relacionados, los que se están desarrollando ampliamente (Batocchio, Ghezzi y Rangone (2016), Buh, Kovačič y Indihar (2015) y Ceribeli, Dallavalle y Merlo (2013). De acuerdo con lo anterior, se plantea la siguiente pregunta: ¿Cuáles serían los factores críticos de éxito y su interrelación en un modelo para implementar el BPM para la cadena de suministro del sector floricultor?

\subsection{Presentación de la empresa}

La empresa objeto de estudio se ubica en el municipio de El Rosal —Cundinamarca-. Fue creada en 1991 con el fin de producir y comercializar flores de alta calidad, donde su actividad principal fue el cultivo de claveles, los que se constituyeron en el producto estrella durante aproximadamente cuatro años. La compañía inicia con 5 ha sembradas, y teniendo en cuenta el aumento de la demanda y el posicionamiento logrado en el mercado, incrementa su infraestructura a 14 ha. Más tarde deja la producción de claveles y se dedica exclusivamente a la producción de rosas tipo exportación. La compañía posee en la actualidad 18 ha de cultivo y su principal destino de exportación es Rusia, a donde va el $82 \%$ de su producción. 


\subsection{Unidades de análisis}

Las unidades sobre las cuales se llevó a cabo el análisis del presente estudio de caso son los procesos correspondientes a la cadena de suministro de la empresa:

- Abastecimiento: hace referencia a todas aquellas actividades relacionadas con la adquisición de materiales, insumos, equipos y demás productos requeridos por la compañía. Incluye la gestión de los inventarios de materiales por utilizar para el proceso de producción. En él se analizan los procesos de gestión de compras, recepción y almacenamiento de materiales y gestión de inventarios.

- Producción: se refiere al desarrollo de planes de producción y a su posterior ejecución. Inicia con la recepción de materiales utilizados para la producción y se encarga de la gestión del inventario de producto en proceso y producto terminado. Este proceso está compuesto a su vez por el proceso de cosecha y poscosecha.

- Entregas: se encarga de la gestión de los pedidos y del transporte o distribución. Procura la gestión del almacenamiento de producto terminado y presta soporte y servicio al cliente. Se analizan los procesos de almacenamiento de producto terminado, facturación y preparación de pedidos y despacho.

\subsection{Método y recolección de la información}

Inicialmente, se utilizaron las bases de datos Science Direct, Emerald, ProQuest, JSTOR y sitios web de corte académico, así como publicaciones de conferencias, de los cuales se obtienen artículos científicos relacionados con la implementación del BPM e identificación de los FCE.

El trabajo de campo se realizó con el fin de recolectar información referente a los procesos estudiados. Se hicieron visitas al cultivo en que, por medio de observación directa, revisión de documentos y preguntas a los responsables de los procesos para comprender sus perspectivas, se obtuvo la información necesaria con el fin de identificar puntos de atención. Además, por medio una entrevista semiestructurada aplicada a la persona responsable del sistema de gestión, se amplió la información sobre el estado actual de la empresa y se construyó el VSM siguiendo la metodología propuesta por Womack y Jones (2011). Con estas fuentes de información, se proporciona validez al estudio, y así poder triangular los elementos identificados. Al mismo tiempo, se siguió una cadena de acciones que aseguró una relación coherente entre la pregunta de investigación y los hallazgos para identificar palabras claves y así categorizar los FCE hallados en la literatura.

\subsection{Análisis de la información}

A partir de los artículos identificados de acuerdo con las palabras claves establecidas para la búsqueda, se hizo una lectura y un análisis de los resúmenes, teniendo en cuenta solo aquellos publicados a partir de 2000, de los cuales se obtuvieron 22 artículos relacionados con identificación, validación y análisis de los FCE en implementaciones del BPM. Cabe aclarar que, de estos 22 artículos, 16 corresponden a publicaciones hechas a partir de 2010. 
Al realizar la lectura completa de estos artículos, se encontró que existe un gran número de FCE, por lo que se decidió utilizar categorías para agruparlos y mostrarlos de forma más sencilla. Se analizó cada categoría con el fin de identificar aquellos que tenían un uso significativo en la literatura por medio del software AQUAD 7 de acceso gratuito, y de acuerdo con los patrones surgidos, se dio forma al modelo que responde a la pregunta de investigación.
Por otra parte, y según la información obtenida en la empresa mediante observación directa y entrevistas, se analizaron las actividades y los tiempos correspondientes a todo el proceso productivo, lo que se validó con su responsable. A partir de esto, se obtuvo información relacionada con el perfil de compras de los materiales utilizados por la empresa, tiempos y cantidades de producción, inventarios y tiempos de entrega de producto, con lo que se estructuraron los procesos de la cadena de suministro.

\section{Resultados y discusión}

$\mathbf{P}$ ara la construcción del modelo, se inició definiendo las categorías de los FCE, apoyadas en dos estudios previos. El primero es el marco teórico propuesto por Trkman (2010), quien argumenta que los FCE pueden ser explicados a partir de la combinación de tres teorías: a) contingencia, b) capacidades dinámicas y c) ajuste entre las tareas y la tecnología. La razón de utilizar esta combinación se basa en el hecho de que adelantar investigaciones en temas relacionados con la gestión, en los que se utilice una estructura simple, hará que posiblemente no se obtengan los resultados esperados (Trkman, 2010).
El segundo es el modelo planteado por Vom Brocke y Rosemann (2010), desarrollado a partir de los modelos de madurez del BPM y en el que se proponen seis elementos críticos del BPM: a) la alineación estratégica, b) la administración, c) los métodos, d) las tecnologías de la información y de la comunicación (TIC), e) la gente y f) la cultura organizacional. Así es que los autores plantean el modelo que se visualiza en la figura 1. 
Figura 1. Modelo propuesto

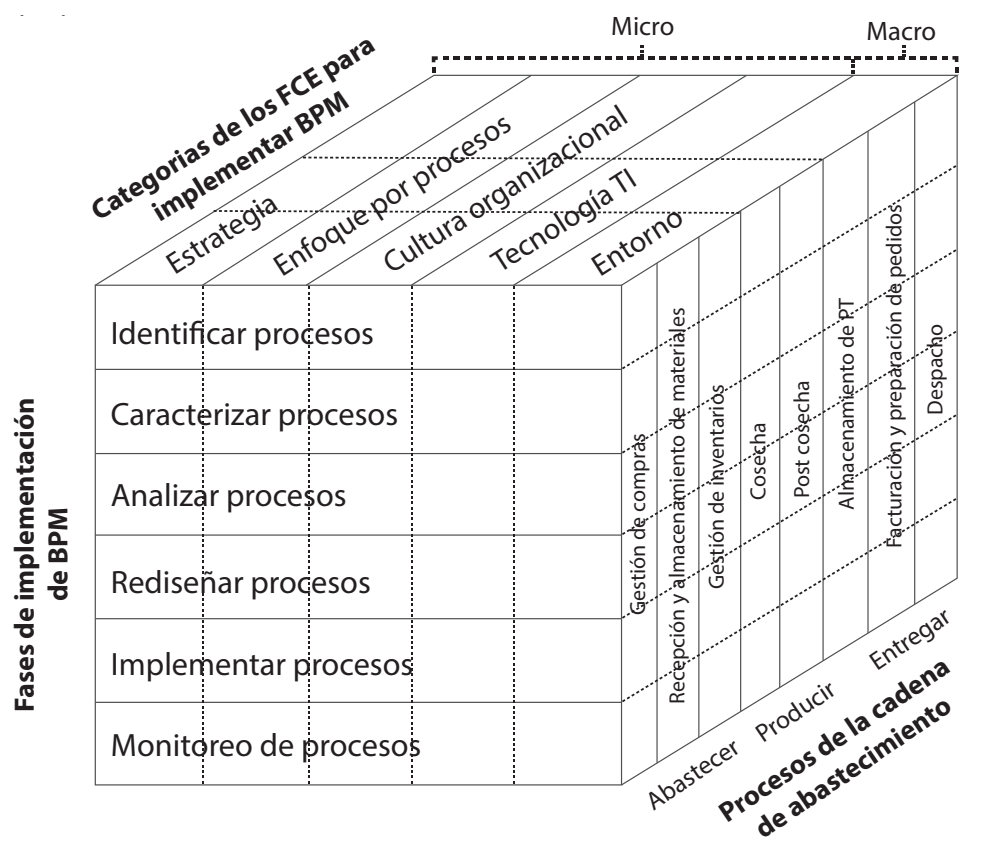

Fuente. Elaboración propia.

Según las dos estructuras citadas, se categorizaron los FCE, que a su vez están agrupados en ambiente micro y ambiente macro. Estas categorías son las siguientes: a) estrategia, b) enfoque por procesos, c) cultura organizacional, d) TI que contienen los FCE correspondientes al ambiente micro y e) entorno que encierra los FCE del ambiente macro. Paso seguido, se identifica la cadena de suministro de la empresa que está compuesta por los procesos de abastecer, producir y entregar (Vitasek, 2013). Por último, el modelo se apoya en las fases de implementación del BPM propuestas por Dumas, La Rosa, Mendling y Reijers (2013).

De acuerdo con lo anterior, se diseñó el modelo basado en tres ejes: a) factores críticos de éxito, b) procesos de la cadena de suministro y c) fases de implementación del BPM, que integra varias herramientas de la ingeniería industrial, como el VSM, el BPM y la SCM. El modelo servirá como marco de referencia para las pymes del sector floricultor, con el que se intenta cubrir aspectos micro y macro de los FCE para lograr una implementación exitosa del BPM.

Se propuso una herramienta de valoración que sirvió de guía para que la empresa identificara aquellos factores más críticos. Esta consiste en una escala por niveles, donde 1 es el más bajo y 3 el más alto. Cada una de estas valoraciones describe el nivel en el que se encuentra la compañía de acuerdo con cada FCE:

- Factores del ambiente micro: hace referencia a aquellos factores que la empresa puede controlar de acuerdo con sus capacidades y gestión de sus recursos.

- Factores del ambiente macro: hace referencia a aquellos factores propios del entorno en el que se desarrolla la empresa, son externos a esta y por consiguiente no puede controlar. 
- Valoración de los FCE: según los hallazgos del estudio de caso, y utilizando la herramienta de valoración propuesta, la figura 2 resume sus resultados.

Figura 2. Valoración de los FCE.

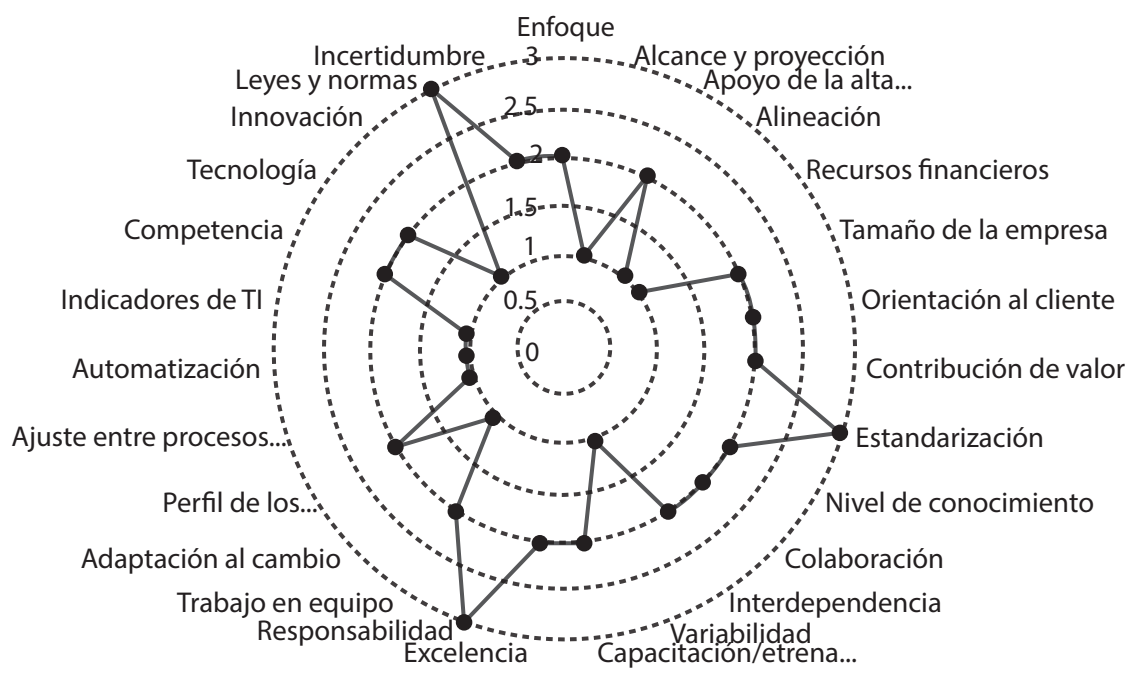

Fuente. Elaboración propia.

La figura 2 muestra los factores críticos; en nivel 1. Factores del ambiente micro: alcance y proyección, alineación, recursos financieros, variabilidad, adaptación al cambio, ajuste entre procesos y TI, automatización e indicadores de TI. Factores del ambiente macro: innovación.
Por otra parte, y de acuerdo con el análisis realizado en el estudio de caso, se identificaron los FCE particulares tanto a las fases de implementación como a la cadena de suministro; estos últimos apoyándose en la herramienta VSM. El modelo propiamente dicho se presenta en la tabla 1. 
Tabla 1. Factores críticos de éxito del ambiente micro

\begin{tabular}{|c|c|c|c|}
\hline \multicolumn{4}{|l|}{ Factores del ambiente micro } \\
\hline \multicolumn{4}{|l|}{ CATEGORÍA: Estrategia } \\
\hline \multicolumn{4}{|l|}{ FCE: Enfoque } \\
\hline Descripción & Nivel 1 & Nivel 2 & Nivel 3 \\
\hline $\begin{array}{l}\text { Se refiere al nivel y/o tipo de mejora } \\
\text { que la compañía desea alcanzar con la } \\
\text { implementación de BPM, el objetivo es } \\
\text { mejorar la eficiencia a través de la adop- } \\
\text { ción de modelos de gestión o, innovar } \\
\text { procesos a través de la introducción de } \\
\text { nuevas tecnologías (vom Brocke, Sch- } \\
\text { miedel, et al., 2015). }\end{array}$ & $\begin{array}{l}\text { No le interesan otros } \\
\text { métodos de gestión o } \\
\text { invertir en tecnología } \\
\text { que permita mejorar su } \\
\text { eficiencia }\end{array}$ & $\begin{array}{l}\text { Satisfacción con el esta- } \\
\text { do actual de su gestión, } \\
\text { pero entiende que debe } \\
\text { mejorar }\end{array}$ & $\begin{array}{l}\text { Desea alcanzar un mayor } \\
\text { nivel de eficiencia refleja- } \\
\text { do en el mejoramiento de } \\
\text { sus procesos de negocio }\end{array}$ \\
\hline \multicolumn{4}{|l|}{ FCE: Alcance y proyección } \\
\hline Descripción & Nivel 1 & Nivel 2 & Nivel 3 \\
\hline $\begin{array}{l}\text { Consiste en definir clara y puntualmen- } \\
\text { te el propósito, necesidades, requeri- } \\
\text { mientos, recursos y duración para llevar } \\
\text { a cabo el proyecto BPM en la compañía, } \\
\text { debe ser compartido, discutido y apro- } \\
\text { bado por las partes interesadas (Box \& } \\
\text { Platss, 2005). }\end{array}$ & $\begin{array}{l}\text { No se existen lineamien- } \\
\text { tos claros ni una proyec- } \\
\text { ción para la ejecución del } \\
\text { proyecto }\end{array}$ & $\begin{array}{l}\text { Se conoce el propósito } \\
\text { del proyecto y existen } \\
\text { lineamientos, pero no } \\
\text { se concreta y se dilata su } \\
\text { aprobación }\end{array}$ & $\begin{array}{l}\text { Existen lineamientos cla- } \\
\text { ros y puntuales, las partes } \\
\text { interesadas las conocen y } \\
\text { están de acuerdo }\end{array}$ \\
\hline \multicolumn{4}{|l|}{ FCE: Apoyo de la alta gerencia } \\
\hline Descripción & Nivel 1 & Nivel 2 & Nivel 3 \\
\hline $\begin{array}{l}\text { Este factor es importante, ya que las } \\
\text { iniciativas de BPM deben contar con } \\
\text { el apoyo total y la participación activa } \\
\text { de los altos directivos de la compañía, } \\
\text { sin esto, el proyecto fracasa (Trkman, } \\
\text { 2010). }\end{array}$ & $\begin{array}{l}\text { No existe apoyo ni inte- } \\
\text { rés por el proyecto }\end{array}$ & $\begin{array}{l}\text { Conoce y apoya el pro- } \\
\text { yecto, pero no se eviden- } \\
\text { cia su participación }\end{array}$ & $\begin{array}{l}\text { Apoyo total a iniciativas } \\
\text { de BPM y participa activa- } \\
\text { mente en el proyecto }\end{array}$ \\
\hline \multicolumn{4}{|l|}{ FCE: Alineación } \\
\hline Descripción & Nivel 1 & Nivel 2 & Nivel 3 \\
\hline $\begin{array}{l}\text { Concordancia que debe existir entre } \\
\text { las estrategias de la organización, los } \\
\text { procesos de la cadena de suministro y } \\
\text { el proyecto BPM, cuyos objetivos deben } \\
\text { ser claros y afines con los de la empresa } \\
\text { (Ravesteyn \& Batenburg, 2010). }\end{array}$ & $\begin{array}{l}\text { No hay alineación entre } \\
\text { la estrategia de la empre- } \\
\text { sa, sus procesos y los ob- } \\
\text { jetivos del proyecto }\end{array}$ & $\begin{array}{l}\text { Baja alineación entre los } \\
\text { objetivos estratégicos de } \\
\text { la empresa, sus procesos } \\
\text { y los objetivos del pro- } \\
\text { yecto }\end{array}$ & $\begin{array}{l}\text { Procesos y objetivos cla- } \\
\text { ros y totalmente alinea- } \\
\text { dos con la estrategia orga- } \\
\text { nizacional }\end{array}$ \\
\hline \multicolumn{4}{|l|}{ FCE: Recursos financieros } \\
\hline Descripción & Nivel 1 & Nivel 2 & Nivel 3 \\
\hline $\begin{array}{l}\text { La compañía debe contar con el pre- } \\
\text { supuesto suficiente para desarrollar y } \\
\text { culminar la implementación de BPM, } \\
\text { de lo contrario se presentarán efectos } \\
\text { negativos en la ejecución de proyectos } \\
\text { que tienen que ver con la adopción de } \\
\text { nuevas tecnologías (Ariyachandra \& } \\
\text { Frolick, 2008). }\end{array}$ & $\begin{array}{l}\text { El presupuesto fue equi- } \\
\text { vocado y no es posible } \\
\text { culminar el proyecto }\end{array}$ & $\begin{array}{l}\text { Se puede culminar el } \\
\text { proyecto, no obstante, el } \\
\text { presupuesto es ajustado }\end{array}$ & $\begin{array}{l}\text { El presupuesto es sufi- } \\
\text { ciente y holgado para cul- } \\
\text { minar el proyecto }\end{array}$ \\
\hline
\end{tabular}


Tabla 1. Factores críticos de éxito del ambiente micro (Continuación)

FCE: Tamaño de la empresa

\begin{tabular}{l|l|l|l}
\hline \multicolumn{1}{|c|}{ Descripción } & Nivel 1 & \multicolumn{1}{c|}{ Nivel 2 } & \\
\hline $\begin{array}{l}\text { Influye no solo en aspectos como la } \\
\text { suficiencia de recursos financieros, hu- } \\
\text { manos o tecnológicos, sino que tam- } \\
\text { bién será más fácil o difícil llegar a un } \\
\text { enfoque en el que se comparta la infor- } \\
\text { mación tal que facilite el diseño de los } \\
\text { procesos logrando la integración y un un } \\
\text { nivel de cumplimiento de los mismos } \\
\text { (Palma-Mendoza \& Neailey, 2015; Pal- } \\
\text { ma-Mendoza et al., 2014). }\end{array}$ & $\begin{array}{l}\text { Influye fuertemente y no } \\
\text { es posible lograr la inte- } \\
\text { gración entre procesos }\end{array}$ & $\begin{array}{l}\text { Es poco relevante pero } \\
\text { no hido fácil compar- } \\
\text { tir la información y lo- } \\
\text { grar la integración entre } \\
\text { procesos }\end{array}$ & $\begin{array}{l}\text { No es relevante y es senci } \\
\text { llo alcanzar el enfoque por } \\
\text { procesos }\end{array}$ \\
\hline
\end{tabular}

FCE: Orientación al cliente

\begin{tabular}{|l|l|l|l}
\hline \multicolumn{1}{|c|}{ Descripción } & \multicolumn{1}{|c|}{ Nivel 1 } & \multicolumn{1}{c|}{ Nivel 2 } & \multicolumn{1}{c}{ Nivel 3} \\
\hline $\begin{array}{l}\text { El cliente es lo más importante y todos } \\
\text { los procesos deben enfocarse a satis- } \\
\text { facer sus necesidades, es un punto de } \\
\text { partida para evidenciar los problemas y y } \\
\text { concientizarse de la necesidad de mejo- } \\
\text { rar (Buh et al., 2015). }\end{array}$ & $\begin{array}{l}\text { No se logra atender to- } \\
\text { dos los requerimientos } \\
\text { del cliente }\end{array}$ & $\begin{array}{l}\text { Se atienden la mayoría } \\
\text { de requerimientos, pero } \\
\text { a un alto costo debido a } \\
\text { ineficiencias en los pro- } \\
\text { cesos }\end{array}$ & $\begin{array}{l}\text { Se atienden todos los re- } \\
\text { querimientos de manera } \\
\text { oportuna y eficiente }\end{array}$ \\
\hline
\end{tabular}

\section{CATEGORÍA: Procesos}

FCE: Contribución de valor

\begin{tabular}{|c|c|c|}
\hline Descripción & Nivel 1 & Nivel 2 \\
\hline $\begin{array}{l}\text { Los resultados de los procesos deben } \\
\text { agregar valor para el cliente, y además } \\
\text { deben ser eficientes, por lo que las orga- } \\
\text { nizaciones utilizan herramientas Lean } \\
\text { para analizar y mejorar sus procesos } \\
\text { (Burlton, 2011). }\end{array}$ & $\begin{array}{l}\text { Procesos ineficientes con } \\
\text { bajo valor agregado }\end{array}$ & $\begin{array}{l}\text { Los procesos son poco } \\
\text { eficientes logrando agre- } \\
\text { gar apenas el suficiente } \\
\text { valor }\end{array}$ \\
\hline
\end{tabular}

\begin{tabular}{|l|l|l|l|}
\hline \multicolumn{1}{|c|}{ Descripción } & \multicolumn{1}{|c|}{ Nivel 1 } & \multicolumn{1}{c|}{ Nivel 2 } & Nivel 3 \\
\hline $\begin{array}{l}\text { Aquellos procesos que son repetitivos } \\
\text { y no requieren altos niveles de conoci- } \\
\text { miento específico, son más fáciles para } \\
\text { lograr la estandarización (vom Brocke, } \\
\text { Schmiedel, et al.,2015). }\end{array}$ & $\begin{array}{l}\text { No existe estandariza- } \\
\text { sos susceptibles de serlo }\end{array}$ & $\begin{array}{l}\text { Existe estandarización } \\
\text { en los procesos, pero no } \\
\text { se cumple a cabalidad }\end{array}$ & $\begin{array}{l}\text { y medidos, se cumplestandarizados } \\
\text { completamente }\end{array}$ \\
\hline
\end{tabular}

Schmiedel, et al., 2015).

FCE: Nivel de conocimiento

\begin{tabular}{|l|l|l|l|}
\hline \multicolumn{1}{|c|}{ Descripción } & Nivel 1 & Nivel 2 & Nivel 3 \\
\hline $\begin{array}{l}\text { Se refiere al conocimiento que la per- } \\
\text { sona posee acerca de los procesos de } \\
\text { negocio y sus aptitudes para desarro- } \\
\text { llarlos, que por lo general, son aquellas } \\
\text { aptitudes blandas que el individuo ha } \\
\text { adquirido y que son necesarias para la } \\
\text { trasferencia de conocimiento entre los } \\
\text { participantes del proceso, por lo que se } \\
\text { requieren altas dosis de juicio humano } \\
\text { para lograrlo (Isik, Mertens, \& Van den } \\
\text { Bergh, 2013). }\end{array}$ & $\begin{array}{l}\text { Desconoce el proceso y } \\
\text { propio criterio }\end{array}$ & $\begin{array}{l}\text { Conoce el proceso, sin } \\
\text { embargo, no comparte } \\
\text { su conocimiento sobre el } \\
\text { mismo }\end{array}$ & $\begin{array}{l}\text { Conoce muy bien el pro- } \\
\text { ceso, lo desarrolla a caba- } \\
\text { lidad y transfiere su cono- } \\
\text { cimiento eficazmente }\end{array}$ \\
\hline
\end{tabular}


Tabla 1. Factores críticos de éxito del ambiente micro (Continuación)

FCE: Colaboración

\begin{tabular}{|c|c|c|}
\hline Descripción & Nivel 1 & Nivel 2 \\
\hline $\begin{array}{l}\text { La colaboración efectiva es el medio } \\
\text { principal para lograr la integración or- } \\
\text { ganizacional con una visión y metas } \\
\text { compartidas, por lo que la generación } \\
\text { de ambientes de comunicación abierta } \\
\text { en los que existe confianza entre com- } \\
\text { pañeros de trabajo, facilita la solución } \\
\text { de problemas bajo un esquema de tra- } \\
\text { bajo en equipo (Esper et al., 2010). }\end{array}$ & $\begin{array}{l}\text { No existe un ambiente } \\
\text { colaborativo entre com- } \\
\text { pañeros y superiores y } \\
\text { no se da una comunica- } \\
\text { ción efectiva entre estos }\end{array}$ & $\begin{array}{l}\text { Hay comunicación, pero } \\
\text { existe el temor de expre- } \\
\text { sar ideas debido a la falta } \\
\text { de confianza entre supe- } \\
\text { riores y subordinados }\end{array}$ \\
\hline
\end{tabular}

FCE: Interdependencia

\begin{tabular}{|c|c|c|c|}
\hline Descripción & Nivel 1 & Nivel 2 & Nivel 3 \\
\hline $\begin{array}{l}\text { Se refiere a la definición de responsabi- } \\
\text { lidades y responsables, donde la inte- } \\
\text { racción entre unidades organizacionales } \\
\text { facilita el intercambio de información } \\
\text { necesario para alcanzar las metas (Jütt- } \\
\text { ner et al., 2010). }\end{array}$ & $\begin{array}{l}\text { No se comparte informa- } \\
\text { ción y apenas se cumple } \\
\text { con las metas organiza- } \\
\text { cionales }\end{array}$ & $\begin{array}{l}\text { Poca interacción, se de- } \\
\text { sarrollan los procesos } \\
\text { con el fin de cumplir me- } \\
\text { tas individuales por área }\end{array}$ & $\begin{array}{l}\text { Se comparte la informa- } \\
\text { ción y se facilita la inte- } \\
\text { racción sabiendo que hace } \\
\text { parte de un sistema }\end{array}$ \\
\hline
\end{tabular}

FCE: Variabilidad

\begin{tabular}{|c|c|c|c|}
\hline Descripción & Nivel 1 & Nivel 2 & Nivel 3 \\
\hline $\begin{array}{l}\text { Son los cambios propios del proceso } \\
\text { pero que afectan el resultado del mis- } \\
\text { mo. En procesos intensivos en conoci- } \\
\text { miento, la variabilidad es un factor que } \\
\text { dificulta el proyecto de BPM, mientras } \\
\text { que los procesos con bajos niveles de } \\
\text { desviación local, se facilita el proyecto } \\
\text { (vom Brocke, Schmiedel, et al., 2015). }\end{array}$ & $\begin{array}{l}\text { Procesos con altos nive- } \\
\text { les de variabilidad y no } \\
\text { existen métricas para su } \\
\text { medición y control }\end{array}$ & $\begin{array}{l}\text { Se intenta controlar la } \\
\text { variabilidad, sistemas de } \\
\text { medición y control inci- } \\
\text { pientes }\end{array}$ & $\begin{array}{l}\text { Se controla la variabilidad } \\
\text { de los procesos y se hacen } \\
\text { estimaciones de la misma }\end{array}$ \\
\hline \multicolumn{4}{|l|}{ CATEGORÍA: Cultura } \\
\hline \multicolumn{4}{|l|}{ FCE: Capacitación y entrenamiento } \\
\hline Descripción & Nivel 1 & Nivel 2 & Nivel 3 \\
\hline $\begin{array}{l}\text { Conocer las implicaciones de su trabajo } \\
\text { y entender cómo los procesos indivi- } \\
\text { duales contribuyen a los objetivos de la } \\
\text { organización es clave para los involucra- } \\
\text { dos en el proyecto, por lo que capacitar } \\
\text { al personal acerca de las ventajas que } \\
\text { trae el enfoque por procesos, facilita a } \\
\text { los colaboradores el cambio de sus mo- } \\
\text { delos mentales tradicionales (Škrinjar } \\
\text { \& Trkman, 2013). }\end{array}$ & $\begin{array}{l}\text { No existen planes de } \\
\text { capacitación y entrena- } \\
\text { miento, el conocimiento } \\
\text { es transmitido de mane- } \\
\text { ra subjetiva entre cola- } \\
\text { boradores }\end{array}$ & $\begin{array}{l}\text { Existen planes de capaci- } \\
\text { tación y entrenamiento, } \\
\text { pero no se aplican juicio- } \\
\text { samente }\end{array}$ & $\begin{array}{l}\text { Existen planes de capa- } \\
\text { citación, entrenamiento } \\
\text { y reentrenamiento un } \\
\text { cronograma definido y } \\
\text { personal calificado para } \\
\text { dirigirlos }\end{array}$ \\
\hline \multicolumn{4}{|l|}{ FCE: Excelencia } \\
\hline Descripción & Nivel 1 & Nivel 2 & Nivel 3 \\
\hline $\begin{array}{l}\text { Se refiere a las actitudes e iniciativas } \\
\text { que tienen las personas y la empresa, } \\
\text { orientadas a la mejoramiento continuo } \\
\text { y la innovación (Schmiedel et al., 2014). }\end{array}$ & $\begin{array}{l}\text { No se cuenta con una } \\
\text { cultura de mejoramiento } \\
\text { continuo }\end{array}$ & $\begin{array}{l}\text { Existe la voluntad, pero } \\
\text { no el compromiso por } \\
\text { generar ideas de mejora- } \\
\text { miento }\end{array}$ & $\begin{array}{l}\text { La compañía promueve la } \\
\text { cultura por mejorar cons- } \\
\text { tantemente y todos están } \\
\text { comprometidos con ella }\end{array}$ \\
\hline
\end{tabular}


Tabla 1. Factores críticos de éxito del ambiente micro (Continuación)

FCE: Responsabilidad

\begin{tabular}{l} 
Descripción \\
\hline Rol que adquieren los implicados en \\
el proyecto y nivel de compromiso de \\
cada uno por el cumplimiento de los \\
objetivos del proceso, lo que ayuda a las \\
personas a identificar su propósito en la \\
empresa y en el proyecto (Box \& Platss, \\
2005).
\end{tabular}

\begin{tabular}{|c|c|} 
& Nivel 1 \\
\hline de & \\
las & No existen roles ni res- \\
ponsabilidades definidas & \\
\hline
\end{tabular}

Cada uno conoce su rol, pero eluden su responsabilidad
Nivel 3

Conoce su rol y está comprometido en el cumplimiento de los objetivos del proceso

FCE: Trabajo en equipo

\begin{tabular}{l|l|l|l}
\multicolumn{1}{c|}{ Descripción } & \multicolumn{1}{|c|}{ Nivel 1 } & \multicolumn{1}{c|}{ Nivel 2 } & \multicolumn{1}{c}{ Nivel 3} \\
\hline $\begin{array}{l}\text { La cultura del trabajo en equipo, así } \\
\text { como las habilidades individuales para } \\
\text { este, son indispensables para lograr la } \\
\text { colaboración inter funcional y por ende } \\
\text { el éxito del proyecto (Ahmad, Francis, } \\
\& \text { Zairi, 2007). }\end{array}$ & $\begin{array}{l}\text { No hay cultura de tra- } \\
\text { bajo en equipo y no hay } \\
\text { colaboración }\end{array}$ & $\begin{array}{l}\text { Se cumple con los ob- } \\
\text { jetivos propuestos, sin } \\
\text { embargo, el trabajo en } \\
\text { equipo no se da constan- } \\
\text { temente }\end{array}$ & $\begin{array}{l}\text { La cultura del trabajo en } \\
\text { equipo está fuertemente } \\
\text { arraigada y se facilita la } \\
\text { colaboración inter-fun- } \\
\text { cional }\end{array}$ \\
\hline
\end{tabular}

FCE: Adaptación al cambio

\begin{tabular}{|l|l|l|l|}
\hline \multicolumn{1}{|c|}{ Descripción } & Nivel 1 & \multicolumn{1}{c|}{ Nivel 2 } & Nivel 3 \\
\hline $\begin{array}{l}\text { Se refiere al temor que las personas } \\
\text { sienten cuando se enfrentan a situacio- } \\
\text { nes fuera de su zona de confort, debido } \\
\begin{array}{l}\text { a la transición tecnológica o la adop- } \\
\text { ción de nuevos modelos mentales que } \\
\text { lleva consigo la gestión por procesos } \\
\text { (Ohtonen \& Lainema, 2011). }\end{array}\end{array}$ & $\begin{array}{l}\text { Alta resistencia al cam- } \\
\text { bio yo se esfuerza por } \\
\text { superarla }\end{array}$ & $\begin{array}{l}\text { Se entiende que se debe } \\
\text { adaptar al cambio, pero } \\
\text { es difícil de lograr }\end{array}$ & $\begin{array}{l}\text { Es más sencillo adaptarse } \\
\text { a los cambios, no obstan- } \\
\text { te, hay que trabajar en ello }\end{array}$ \\
\hline
\end{tabular}

FCE: Perfil de los funcionarios

\begin{tabular}{|l|l|l|l|}
\hline \multicolumn{1}{|c|}{ Descripción } & \multicolumn{1}{|c|}{ Nivel 1 } & \multicolumn{1}{c|}{ Nivel 2 Nivel 3 } \\
\hline $\begin{array}{l}\text { Es importante generar en las personas } \\
\text { el deseo por desarrollar competencias y } \\
\text { habilidades para que animen faciliten } \\
\text { la adaptación de los cambios necesarios } \\
\text { (Bandara et al., 2005). }\end{array}$ & $\begin{array}{l}\text { Fuerte arraigo a sus } \\
\text { modelos mentales. No } \\
\text { se hace nada para cam- } \\
\text { biarlo }\end{array}$ & $\begin{array}{l}\text { Se entiende que se debe } \\
\text { adaptar al cambio, pero } \\
\text { es difícil de lograr }\end{array}$ & $\begin{array}{l}\text { Se diseñan y desarrollan } \\
\text { planes que facilitan la } \\
\text { adopción de los cambios }\end{array}$ \\
\hline
\end{tabular}

CATEGORÍA: Tecnologías de la información

FCE: Ajuste entre procesos y TI

\begin{tabular}{|l|l|l|l|}
\hline \multicolumn{1}{|c|}{ Descripción } & \multicolumn{1}{|c|}{ Nivel 1 } & \multicolumn{1}{c|}{ Nivel 2 } & Nivel 3 \\
\hline $\begin{array}{l}\text { Consiste en disminuir la brecha que hay } \\
\text { entre la utilización de la tecnología y la } \\
\text { ejecución de los procesos de negocio, } \\
\text { sin embargo, inversiones en TI no siem- } \\
\text { pre son posibles, por lo que es deber de } \\
\text { la alta gerencia analizar esta decisión, ya } \\
\text { que la tecnología por sí sola, no implica } \\
\text { una mejora (Trkman, 2010). }\end{array}$ & $\begin{array}{l}\text { No cuenta con in- } \\
\text { obsoleta para ejecutar } \\
\text { los procesos }\end{array}$ & $\begin{array}{l}\text { La infraestructura de TI } \\
\text { no es suficiente o ade- } \\
\text { cuada y soporta acepta- } \\
\text { blemente los procesos }\end{array}$ & $\begin{array}{l}\text { La infraestructura de TI es } \\
\text { suficiente y adecuada para } \\
\text { soportar los procesos }\end{array}$ \\
\hline
\end{tabular}


Tabla 1. Factores críticos de éxito del ambiente micro (Continuación)

FCE: Automatización

\begin{tabular}{|c|c|c|c|}
\hline Descripción & Nivel 1 & Nivel 2 & Nivel 3 \\
\hline $\begin{array}{l}\text { La automatización de procesos se refie- } \\
\text { re a la utilización de las TI para apoyar o } \\
\text { reemplazar la ejecución de aquellos pro- } \\
\text { cesos que son repetitivos o rutinarios, } \\
\text { sin embargo, automatizar puede ser } \\
\text { costoso y tener limitaciones propias de } \\
\text { la tecnología a utilizar (Trkman, 2010). }\end{array}$ & $\begin{array}{l}\text { No se cuenta con proce- } \\
\text { sos automatizados. }\end{array}$ & $\begin{array}{l}\text { Algunos procesos re- } \\
\text { petitivos se encuentran } \\
\text { automatizados }\end{array}$ & $\begin{array}{l}\text { Todos los procesos rutina } \\
\text { rios se encuentran auto } \\
\text { matizados }\end{array}$ \\
\hline \multicolumn{4}{|l|}{ FCE: Indicadores de TI } \\
\hline Descripción & Nivel 1 & Nivel 2 & Nivel 3 \\
\hline $\begin{array}{l}\text { Son útiles para identificar falencias en } \\
\text { el desarrollo del proyecto, así como los } \\
\text { avances en el mismo. Es importante } \\
\text { establecerlos y nombrar a los respon- } \\
\text { sables de su cumplimiento (Buh et al., } \\
\text { 2015). }\end{array}$ & $\begin{array}{l}\text { No hay indicadores } \\
\text { definidos }\end{array}$ & $\begin{array}{l}\text { Existen indicadores, sin } \\
\text { embargo, no se miden de } \\
\text { manera juiciosa }\end{array}$ & $\begin{array}{l}\text { Existe un sistema de in } \\
\text { dicadores, se miden y } \\
\text { toman acciones sobre su } \\
\text { resultados }\end{array}$ \\
\hline
\end{tabular}

Factores del ambiente macro

\section{CATEGORÍA: Entorno}

FCE: Competencia

\begin{tabular}{|c|c|c|c|}
\hline Descripción & Nivel 1 & Nivel 2 & Nivel 3 \\
\hline $\begin{array}{l}\text { Hace referencia a la cantidad de empre- } \\
\text { sas y a su capacidad para competir entre } \\
\text { sí dentro de una industria o sector, se } \\
\text { busca tener claridad de la competitivi- } \\
\text { dad de la organización y su situación ac- } \\
\text { tual para poder comparar la mejora que } \\
\text { se da al implementar BPM (Imanipour, } \\
\text { Talebi, \& Rezazadeh, 2012). }\end{array}$ & $\begin{array}{l}\text { No se tiene claridad } \\
\text { acerca de su ventaja } \\
\text { competitiva. Se encuen- } \\
\text { tra en un nivel inferior al } \\
\text { de sus competidores }\end{array}$ & $\begin{array}{l}\text { Se tiene alguna claridad } \\
\text { acerca de su ventaja com- } \\
\text { petitiva. Se encuentra al } \\
\text { mismo nivel que el de } \\
\text { sus competidores }\end{array}$ & $\begin{array}{l}\text { Total, claridad acerca de } \\
\text { su ventaja competitiva. } \\
\text { Se encuentra en un nivel } \\
\text { superior al de sus compe- } \\
\text { tidores }\end{array}$ \\
\hline \multicolumn{4}{|l|}{ FCE: Tecnología } \\
\hline Descripción & Nivel 1 & Nivel 2 & Nivel 3 \\
\hline $\begin{array}{l}\text { Oferta de productos referentes a hard- } \\
\text { ware, software, herramientas BPM y } \\
\text { acompañamiento ofrecido, que están } \\
\text { presentes en el mercado (Ahmad et al., } \\
\text { 2007; Imanipour et al., 2012). }\end{array}$ & $\begin{array}{l}\text { Desconoce totalmente } \\
\text { la oferta de tecnología } \\
\text { posible a utilizar en su } \\
\text { negocio }\end{array}$ & $\begin{array}{l}\text { Conoce en cierta medida } \\
\text { la oferta de tecnología } \\
\text { posible a utilizar en su } \\
\text { negocio. No está seguro } \\
\text { de su utilización }\end{array}$ & $\begin{array}{l}\text { Conoce la oferta de tecno- } \\
\text { logía posible a utilizar en } \\
\text { su negocio y está dispues- } \\
\text { to a utilizarla }\end{array}$ \\
\hline \multicolumn{4}{|l|}{ FCE: Innovación } \\
\hline Descripción & Nivel 1 & Nivel 2 & Nivel 3 \\
\hline $\begin{array}{l}\text { Análisis sistemático de los cambios } \\
\text { tecnológicos para transformarlos en } \\
\text { oportunidades de negocio, realizar la } \\
\text { creación o el desarrollo de nuevos pro- } \\
\text { ductos y/o servicios, la modificación de } \\
\text { un producto y la mejora de los procesos } \\
\text { para producirlos, nuevas fórmulas de } \\
\text { comercialización, y su introducción en } \\
\text { un mercado. No es un evento aislado } \\
\text { sino la respuesta continua a circunstan- } \\
\text { cias cambiantes (Mircea et al., 2016). }\end{array}$ & $\begin{array}{l}\text { Se queda rezagado ante } \\
\text { los cambios del entorno } \\
\text { y no genera oportunida- } \\
\text { des de negocio acordes } \\
\text { con los cambios }\end{array}$ & $\begin{array}{l}\text { Entiende que el entorno } \\
\text { es cambiante, pero se } \\
\text { demora en generar nue- } \\
\text { vas oportunidades de } \\
\text { negocio acordes con los } \\
\text { cambios }\end{array}$ & $\begin{array}{l}\text { Está en línea con los } \\
\text { cambios tecnológicos, del } \\
\text { mercado y del entor- } \\
\text { no, convirtiéndolos en } \\
\text { oportunidades para la } \\
\text { innovación empresarial }\end{array}$ \\
\hline
\end{tabular}


Tabla 1. Factores críticos de éxito del ambiente micro (Continuación)

\begin{tabular}{|c|c|c|c|}
\hline \multicolumn{4}{|l|}{ FCE: Leyes y normas } \\
\hline Descripción & Nivel 1 & Nivel 2 & Nivel 3 \\
\hline $\begin{array}{l}\text { Condiciones normativas del país que } \\
\text { promueven el desarrollo empresarial, } \\
\text { protegen la industria nacional, impul- } \\
\text { san el acceso a nuevos mercados y velan } \\
\text { por la preservación del medio ambiente. } \\
\text { (Anexo B. Normatividad que rige al sec- } \\
\text { tor floricultor) }\end{array}$ & $\begin{array}{l}\text { Desconoce en su totali- } \\
\text { dad la normatividad del } \\
\text { país, así como los conve- } \\
\text { nios para acceder a otros } \\
\text { mercados }\end{array}$ & $\begin{array}{l}\text { Conoce en cierta medida } \\
\text { la normatividad del país } \\
\text { y sabe de la existencia de } \\
\text { convenios para acceder a } \\
\text { otros mercados }\end{array}$ & $\begin{array}{l}\text { Conoce bien la normativi- } \\
\text { dad del país y aprovecha } \\
\text { los convenios para acce- } \\
\text { der a otros mercados }\end{array}$ \\
\hline \multicolumn{4}{|l|}{ FCE: Incertidumbre política y económica } \\
\hline Descripción & Nivel 1 & Nivel 2 & Nivel 3 \\
\hline $\begin{array}{l}\text { Se refiere a las condiciones económicas } \\
\text { y políticas que permiten a las compa- } \\
\text { ñías desarrollar sus planes en el largo } \\
\text { plazo (vom Brocke, Schmiedel, et al., } \\
2015) \text {. }\end{array}$ & $\begin{array}{l}\text { Desconoce en su totali- } \\
\text { dad las condiciones eco- } \\
\text { nómicas y políticas del } \\
\text { país. Toma decisiones } \\
\text { atrasadas según las cir- } \\
\text { cunstancias }\end{array}$ & $\begin{array}{l}\text { Conoce en cierta medida } \\
\text { las condiciones económi- } \\
\text { cas y políticas del país. } \\
\text { Toma decisiones sobre la } \\
\text { marcha de los aconteci- } \\
\text { mientos }\end{array}$ & $\begin{array}{l}\text { Conoce bien las condicio- } \\
\text { nes económicas y políti- } \\
\text { cas del país. Se informa y } \\
\text { toma decisiones en pro- } \\
\text { cura de adelantarse a las } \\
\text { circunstancias }\end{array}$ \\
\hline
\end{tabular}

Fuente. Elaboración propia.

\section{Conclusiones y trabajos futuros}

Se examinó la literatura relacionada con los $\checkmark$ factores críticos de éxito que afectan las implementaciones del BPM. Con el estudio de caso, se identificaron aquellos que afectan tanto las diferentes etapas de implementación como los procesos de la cadena de suministro.

Algunos autores presentan enfoques similares en los que argumentan que se debe prestar atención al efecto que causan estos FCE en proyectos de implementación de la Enterprise Resource Planning (ERP) (Ram y Corkindale, 2012) o en proyectos que conlleven la orientación por procesos y la gestión de cadenas de suministro (McCormack y Johnson, 2001). Se encontraron similitudes en las definiciones referidas a los FCE dadas por los diferentes autores; sin embargo, no hacen una integración entre los procesos de la cadena de suministro y la implementación del BPM.
También, se encontró que en la literatura hay carencia de estudios que validen de manera experimental la influencia de los FCE, por lo que deberían ser analizados con mayor detenimiento (Buh, Kovačič y Indihar, 2015; Ram y Corkindale, 2012; Trkman, 2010). Soportado en esto, se diseñó un modelo en el que se analiza la interacción que hay entre los FCE, los procesos de la cadena de suministro y las fases de implementación del BPM, donde se encontró que los FCE no son los mismos para las etapas de implementación ni para cada uno de los procesos de la cadena de suministro. Además, los FCE descritos apuntan en su mayoría a revisar la alineación entre los objetivos estratégicos de la empresa y los procesos de su cadena de suministro.

Teniendo presente los resultados obtenidos, se puede concluir que los FCE correspondientes a la categoría Estrategia son a los que más atención se debe prestar si la empresa deseara 
implementar el BPM, razón por la que se deben adelantar acciones para lograr un enfoque por procesos dirigido a mejorar su sistema logístico.

Estos resultados se convierten en una guía para identificar aquellos FCE relacionados con la cadena de suministro de un cultivo de flores, que está dirigida a empresas que deseen adelantar proyectos del BPM, por lo que es una herramienta útil que permite enfocarse en un conjunto de FCE y así poder dirigir los esfuerzos hacia estos en particular y lograr con éxito la implementación.

Las empresas del sector floricultor colombiano no son lo suficientemente eficientes y se enfrentan a un gran reto como es la consolidación de sus procesos (Sepúlveda, 2014). Identificar los FCE que afectan las implementaciones del BPM permite alinear sus objetivos estratégicos con los procesos de su cadena de suministro. De esta manera, se puede ver que el BPM es una herramienta útil para que las organizaciones alcancen sus metas, logren el compromiso de las personas y gestionen de manera eficiente sus recursos.
Otras investigaciones concernientes pueden estar dirigidas a estudiar la correlación que hay entre los FCE y los procesos de la cadena de suministro de acuerdo con las fases de implementación del BPM. De manera similar, pueden adelantarse estudios que integren análisis estadísticos que prueben la influencia de los FCE sobre el desempeño de la cadena, aplicando la herramienta en varias empresas.

Una oportunidad de investigación puede estar dirigida a identificar casos de éxito y casos fallidos en empresas del sector floricultor en implementaciones del BPM, para cruzar los resultados con el fin de encontrar similitudes y compartir lecciones aprendidas que conlleven mejorar el conocimiento sobre este tema.

El modelo establecido propuso un marco de referencia para futuras investigaciones que permitan hacer un diagnóstico y determinar el nivel de los FCE por tener en cuenta en proyectos de implementación de modelos BPM. Con esta calificación se puede establecer el camino a seguir por parte de la empresa para subir al siguiente nivel, e ir mejorando los puntos claves para el desarrollo y la implementación del BPM.

\section{Referencias}

Ahmad, H., Francis, A. y Zairi, M. (2007). Business process reengineering: Critical success factors in higher education. Business Process Management Journal, 13(3), 451-469.

Al-Mashari, M. y Zairi, M. (1999). BPR implementation process: An analysis of key success and failure factors. Business Process Management Journal, 5(1), 87-112.

Antonucci, Y., Bariff, M., Benedict, T., Champlin, B., Downing, B., Franzen, J., Madison, D., Lusk, S., Spanyi, A., Treat, M., Zhao, L., \& Raschke, R., (2009). Business Process Management Common Body Of Knowledge (Second). Chicago: ABPMP.
Ariyachandra, T. R. y Frolick, M. N. (2008). Critical success factors in business performance management: Striving for success. Information Systems Management, 25(2), 113-120.

Armistead, C., Pritchard, J.-P. y Machin, S. (1999). Strategic business process management for organisational effectiveness. Long Range Planning, 32(1), 96-106.

Asikhia, U. O. y Awolusi, D. O. (2015). Assessment of critical success factors of business process re-engineering in the Nigerian oil and gas industry. South African Journal of Business Management, 46(2), 1-14. 
Asociación Colombiana de Exportadores de Flores. (2017). Cifras estadísticas. Recuperado de www.asocolflores. org/servicios/cifras-estadisticas/36

Bai, C. y Sarkis, J. (2013). A grey-based DEMATEL model for evaluating business process management critical success factors. International Journal of Production Economics, 146(1), 281-292.

Bandara, W., Alibabaei, A. y Aghdasi, M. (2009). Means of achieving business process management success factors. Ponencia presentada en Proceedings of the 4th Mediterranean Conference on Information Systems, Atenas.

Bandara, W., Gable, G. G. y Rosemann, M. (2005). Factors and measures of business process modelling: Model building through a multiple case study. European Journal of Information Systems, 14(4), 347-360.

Batocchio, A., Ghezzi, A. y Rangone, A. (2016). A method for evaluating business models implementation process. Business Process Management Journal, 22(4), 712-735.

Bider, I. y Perjons, E. (2015). Design science in action: developing a modeling technique for eliciting requirements on business process management (BPM) tools. Software \& Systems Modeling, 14(3), 1159-1188.

Bosilj Vukšić, V., Milanović Glavan, L. y Suša, D. (2015). The role of process performance measurement in BPM adoption outcomes in Croatia. Economic and Business Review, 17(1), 117-143.

Box, S. y Platss, K. (2005). Business process management: Establishing and maintaining project aligment. Business Process Management Journal, 11(4), 370-387.

Buh, B., Kovačič, A. y Indihar Štemberger, M. (2015). Critical success factors for different stages of business process management adoption: A case study. Economic Research-Ekonomska Istraživanja, 28(1), 243-258.

Burlton, R. (2011). BPM critical success factors lessons learned from successful BPM organizations. Business Rules Journal, 12(10), 1-6.

Ceribeli, H. B., Dallavalle de Pádua, S. I. y Merlo, E. M. (2013). BPM: um estudo de caso dos fatores críticos de sucesso. Globalization, Competitiviness \& Governability, 7(2), 106-117.

CSCMP Supply Chain Management Definitions and Glossary. Recuperado de http://cscmp.org/CSCMP/ Educate/SCM_Definitions_and_Glossary_of_Terms/ CSCMP/Educate/SCM_Definitions_and_Glossary_ of_Terms.aspx?hkey $=\overline{6} 0879588-\mathrm{f} \overline{6} 5 \mathrm{f}-\overline{4} \mathrm{ab} 5-8 \mathrm{c} 4 \mathrm{~b}$ 6878815 ef921

Crhistopher, M. (2011). Logistics and supply chain management: Creating value-adding networks (4. ${ }^{\mathrm{a}}$ ed.). Edimburgh: Pearson Education.
Dallavalle de Pádua, S. I., Hornos da Costa, J., Segatto, M., de Souza Júnior, M. A. y Jabbour, C. J. C. (2014). BPM for change management: Two process diagnosis techniques. Business Process Management Journal, 20(2), 247-271.

Departamento Administrativo Nacional de Estadística. (2017, mayo 3). Boletín Técnico. Recuperado de https:// www.dane.gov.co/files/investigaciones/boletines/ exportaciones/bol_exp_mar17.pdf

Dumas, M., La Rosa, M., Mendling, J. y Reijers, H. A. (2013). Fundamentals of business process management. Heidelberg: Springer.

Esper, T. L., Defee, C. C. y Mentzer, J. T. (2010). A framework of supply chain orientation. The International Journal of Logistics Management, 21(2), 161-179.

Galvis-Lista, E. A. y González-Zabala, M. P. (2014). Herramientas para la gestión de procesos de negocio y su relación con el ciclo de vida de los procesos de negocio: una revisión de literatura. Ciencia e ingeniería Neogranadina, 24(2), 37-55.

Ghalimi, I. y McGoveran, D. (2004). Standards and BPM. Business Integration Journal, 1, 16-18. Recuperado de http:// gcj.alternativetech.com/publications/BIJ/BPM-\%20 Standards\%20and\%20BPM\%20ghalimi\%20april04.pdf

González Cárdenas, A. C. (2013). Intercambio de información en las cadenas de suministro internacionales. Serie Comercio Internacional, 120.

González, A. y Sarmiento, Z. (2014). Optimización de las operaciones logísticas de la cadena de exportación de flores. Flor y Cultura Colombiana, 10-19.

Hammer, M. (2002). Process management and the future of Six Sigma. MIT Sloan Management Review, 43 (2), 26-32.

Hernández-Nariño, A., Delgado-Landa, A., Marqués-León, M., Nogueira-Rivera, D., Medina-León, A. y Negrín-Sosa, E. (2016). Generalización de la gestión por procesos como plataforma de trabajo de apoyo a la mejora de organizaciones de salud. Gerencia y Políticas de Salud, 15(31), 66-87.

Imanipour, N., Talebi, K. y Rezazadeh, S. (2012). Obstacles in business process management (BPM) implementation and adoption in SMEs. Recuperado de https://papers.ssrn. com/sol3/papers.cfm?abstract_id=1990609

Işik, Ö., Mertens, W. y Van den Bergh, J. (2013). Practices of knowledge intensive process management: quantitative insights. Business Process Management Journal, 19(3), 515-534.

Jüttner, U., Christopher, M. y Godsell, J. (2010). A strategic framework for integrating marketing and supply chain strategies. The International Journal of Logistics Management, 21(1), 104-126. 
Kohlbacher, M. y Gruenwald, S. (2011). Process orientation: Conceptualization and measurement. Business Process Management Journal, 17(2), 267-283.

McCormack, K. y Johnson, B. (2001). Business process orientation, supply chain management, and the e-corporation. IIE Solutions, 33(10), 33-33.

Mentzer, J. T., DeWitt, W., Keebler, J. S., Min, S., Nix, N. W., Smith, C. D. y Zacharia, Z. G. (2001). Defining supply chain management. Journal of Business Logistics, 22(2), 1-25.

Min, S. y Mentzer, J. T. (2004). Developing and measuring supply chain management concepts. Journal of Business Logistics, 25(1), 63-99.

Mircea, M., Ghilic-Micu, B., Stoica, M. y Sinioros, P. (2016). Inter-organizational performance and business process management in collaborative networks. Economic Computation and Economic Cybernetics Studies and Research, 50(2), 107-123.

Morais, R. M. de, Kazan, S., Pádua, S. I. D. de y Costa, A. L. (2010). An analysis of BPM lifecycles: From a literature review to a framework proposal. Business Process Management Journal, 20(3), 412-432.

Munive-Hernández, E. J., Dewhurst, F. W., Pritchard, M. C. y Barber, K. D. (2004). Modelling the strategy management process: An initial BPM approach. Business Process Management Journal, 10(6), 691-711.

Münstermann, B., Eckhardt, A. y Weitzel, T. (2010). The performance impact of business process standardization. Business Process Management Journal, 16(1), 29-56.

Ohtonen, J. y Lainema, T. (2011). Critical success factors in business process management: A literature review. Recuperado de https://s3.amazonaws.com/academia. edu.documents/31319485/Critical_key_factors. pdf? AWSAccessKeyId= AKIAIWOWȲYGZ̄2Y53UL3A\&Expires $=1536966168 \&$ Signature $=$ Q05SGIxIXvijblppXGfeyXu4OhI\%3D\&response-content-disposition=inline\%3B\%20filename\%3DCritical_key_factors. pdf

Ozcelik, Y. (2010). Do business process reengineering projects payoff? Evidence from the United States. International Journal of Project Management, 28(1), 7-13.

Palma-Mendoza, J. A. y Neailey, K. (2015). A business process re-design methodology to support supply chain integration: Application in an Airline MRO supply chain. International Journal of Information Management, 35(5), 620-631.

Palma-Mendoza, J. A., Neailey, K. y Roy, R. (2014). Business process re-design methodology to support supply chain integration. International Journal of Information Management, 34(2), 167-176.
Pantoja, M. C. y Donoso, E. (2015). Optimización de las operaciones logísticas de la cadena de exportación de flores. Recuperado de http://asocolflores.net.co/comunicaciones/revistas/floricultura-colombiana-no-6/22/5

Porter, M. E. (1985). Competitive advantage, creating and sustaining superior performance. Nueva York, NY: Simon and Schuster Inc.

Quirós, M. L. (2001). La floricultura en Colombia en el marco de la globalización: aproximaciones hacia un análisis micro y macroeconómico. Revista Universidad EAFIT, 37(122), 59-68.

Ram, J. y Corkindale, D. (2012). How "critical" are the critical succes factors (CSFs)? Examining the role of CSFs for ERP. Business Process Management Journal, 20(1), 151-174.

Ravesteyn, P. y Batenburg, R. (2010). Surveying the critical success factors of BPM $\square$ systems implementation. Business Process Management Journal, 16(3), 492-507.

Rodríguez, C. (2015). Qué es Business Process Management (BPM): definiciones y conceptos. Revista de la Escuela Colombiana de Ingeniería, 25(98), 23-29.

Rodríguez, C., Pérez, A. y Rodríguez, J. (2015). Modelos estratégicos para la implementación del BPM en pymes de Colombia. Bogotá.

Schmiedel, T., Vom Brocke, J. y Recker, J. (2014). Development and validation of an instrument to measure organizational cultures' support of business process management. Information \& Management, 51 (1), 43-56.

Sepúlveda, P. (2014). Análisis de eficiencia técnica y estudio de casos en los cultivos de flores de la sabana de Bogotá (Colombia). Pensamiento y Gestión, 36(1), 289-323.

Škrinjar, R. y Trkman, P. (2013). Increasing process orientation with business process management: Critical practices. International Journal of Information Management, 33(1), 48-60.

Trkman, P. (2010). The critical success factors of business process management. International Journal of Information Management, 30(2), 125-134.

Trkman, P., McCormack, K., Valadares De Oliveira, M. y Bronzo, M. (2010). The impact of business analytics on supply chain performance. Decision Support Systems, 49(3), 318-327.

Vitasek, K. (2013). Supply Chain Management. Terms and Glossary. Recuperado de https://bit.ly/2DWAwvA.

Vom Brocke, J. y Rosemann, M. (2010). Handbook on business process management. Heidelberg: Springer.

Vom Brocke, J., Zelt, S. y Schmiedel, T. (2015a). Considering context in business process management: The BPM context framework. BPM Trends, 1, 272. 
Vom Brocke, J., Zelt, S. y Schmiedel, T. (2015b). On the role of context in business process management. International Journal of Information Management, 36(3), 486-495.

Womack, J. P. y Jones, D. T. (2011). Seeing the whole value stream (2. ${ }^{\mathrm{a}}$ ed.). Cambridge: Lean Enterprise Institute.

Wong, W. P. (2013). Business-process management: a proposed framework for future research. Total Quality Management $\mathcal{E}$ Business Excellence, 24(5-6), 719-732.

Yin, R. K. (2003). Case study research: Design and methods (3. ${ }^{\mathrm{a}}$ ed.). Thousand Oaks: SAGE Publications.

Zairi, M. (1997). Business process management: A boundaryless approach to modern competitiveness. Business Process Management Journal, 3(1), 64-80. 